\title{
Variabilidade atmosférica da precipitação associada com as circulações de brisas marítimas e terrestres no nordeste do estado do Pará, Brasil Atmospheric variability in precipitation associated with on-shore and off-shore breezes in northeastern Pará
}

\author{
Michell Fontenelle Germano', Maria Isabel Vitorino"l, Gabriel Brito Costa"II, \\ Adriano Marlison Leão de Souza ${ }^{\mathrm{IV}}$, Jefferson Inayan de Oliveira Souto"I \\ Instituto Nacional de Pesquisas Espaciais. São José dos Campos, São Paulo, Brasil \\ "Universidade Federal do Pará. Belém, Pará, Brasil \\ IIIUniversidade Federal do Oeste do Pará. Santarém, Pará, Brasil \\ ^vniversidade Federal Rural da Amazônia. Belém, Pará, Brasil
}

\begin{abstract}
Resumo: As circulações de brisas marítimas e terrestres e o seu papel na precipitação no litoral paraense são amplamente investigados neste estudo. Para isso, foram coletados dados de vento (direção e velocidade) e precipitação, em uma frequência de dez minutos, a partir da torre micrometeorológica localizada no sítio experimental da Universidade Federal Rural da Amazônia (UFRA), em Cuiarana, município de Salinopólis, Pará. Além disso, foram utilizadas imagens de satélite feitas no canal visível do Geostationary Operational Environmental Satellite (GOES), bem como dados do CPC MORPHing technique (CMORPH), para a visualização espacial da precipitação. Os resultados demonstram que as circulações de brisas são mais intensas durante o período chuvoso (março) em relação ao período menos chuvoso (agosto). A precipitação local observada, que pode estar relacionada com a brisa marítima, apresentou maior frequência durante o final da tarde e o início da noite. Estes resultados permitem evidenciar a influência das brisas na precipitação local.
\end{abstract}

Palavras-chave: Circulações locais. Precipitação. Brisas. Amazônia.

Abstract: Sea (onshore) and land (offshore) breezes and their role in precipitation on the coast of Pará were investigated. We retrieved wind data (direction and velocity) and precipitation at 10 minute intervals from the micrometeorological tower located at the Universidade Rural da Amazônia UFRA experimental site at Cuiarana in the municipality of Salinópolis, Pará state. We used satellite images from the Geostationary Operational Environmental Satellite (GOES) satellite in the visible channel and CPC MORPHing technique $(\mathrm{CMORPH})$ data for spatial visualization of precipitation. Results show that the breezes are more intense during the rainy season (March) than during the dry season (August). Observed local precipitation, which may be related to sea breezes, showed a higher frequency during the late afternoon and early evening. These results allow us to evaluate the influence of sea breezes on costal local precipitation.

Keywords: Local circulation. Precipitation. Breezes. Amazon.

GERMANO, M. F., M. I. VITORINO, G. B. COSTA, A. M. L. SOUZA \& J. I. O. SOUTO, 2017. Variabilidade atmosférica da precipitação associada com as circulações de brisas marítimas e terrestres no nordeste do estado do Pará, Brasil. Boletim do Museu Paraense Emílio Goeldi. Ciências Naturais 11(3): 303-312.

Autor para correspondência: Michell Fontenelle Germano. Instituto Nacional de Pesquisas Espaciais. Programa de Pós-Graduação em Meteorologia. Av. dos Astronautas, 1.758 - Jardim da Granja. São José dos Campos, SP, Brasil. CEP 12227-010 (michellfgermano@gmail.com). Recebido em 25/02/2015

Aprovado em 27/03/2017

Responsabilidade editorial: Maria de Lourdes Pinheiro Ruivo e Rogério Rosa da Silva

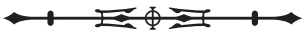




\section{INTRODUÇÃO}

A circulação de brisas é um fenômeno bem conhecido na literatura, cuja importância se deve principalmente ao fato de exercer influência na precipitação diurna em regiões costeiras (Kousky, 1980; Negriet al., 1994; Germano et al., 2017). A brisa marítima (BM) inicia-se devido a um gradiente de temperatura existente entre $\mathrm{o}$ ar, entre $\mathrm{O}$ continente ( $\mathrm{Ta}$ ) e o oceano (Tb). Esta diferença torna-se positiva após o nascer do sol e alcança o máximo em torno de duas horas após o meio dia, diminuindo novamente durante a tarde, até chegar a valores negativos durante a madrugada (Haurwitz, 1947).

Na contramão da brisa marítima, há a brisa terrestre (BT), a qual, por sua vez, não tem sido tão estudada quanto à primeira, devido à pouca influência que exerce sobre chuvas continentais, pois é esperado que a BT favoreça a precipitação sobre os oceanos (Germano et al., 2017). Teixeira (2008), em estudo observacional realizado no município de Fortaleza, no estado do Ceará, Brasil, constatou que a circulação de brisa terrestre está associada, em geral, com o aumento da frequência de chuvas durante a madrugada e o início da manhã, porém tais chuvas tendem a ser mais rápidas e com pouca intensidade.

Kousky (1980) mostrou a importância da circulação da brisa marítima nas regiões Norte e Nordeste do Brasil. Em seus resultados, este pesquisador constatou que a influência da frente de brisa fica clara devido ao horário de ocorrência da precipitação no continente, entre 9:00-15:00 - hora local $(\mathrm{HL})$-, durante o período de desenvolvimento da brisa marítima. No entanto, durante o período da noite, este fluxo se opõe à circulação da brisa terrestre, produzindo uma zona de convergência ao longo da costa (Kousky, 1980). Kousky (1980) também identificou que, em Soure, a precipitação ocorre mais cedo do que em Belém, devido à proximidade do local em relação ao oceano, experimentando máximo de precipitação durante o final da tarde, período em que a frente de brisa está se formando. Janowiak et al. (2005) demonstraram, por meio de estimativas de precipitação com o uso da técnica $\mathrm{CMORPH}$, que o ciclo diurno de precipitação é altamente modulado pela circulação de BM. Além disso, estes pesquisadores também comprovaram que a BM produz máximo noturno em áreas com até $500 \mathrm{~km}$ continente adentro.

As linhas de instabilidade (LI) amazônicas também dependem da circulação de BM. Cohen et al. (1995), em estudo observacional, citaram que a formação das LI amazônicas dependem diretamente de BM. Este mecanismo atua como gatilho para a formação de nuvens convectivas, devido à advecção de umidade produzida pelas brisas. Sun \& Orlanski (1981) avaliaram, através de modelo linear, que as LI, associadas à instabilidade trapezoidal, podem facilmente ser iniciadas pela BM e se propagar quilômetros por continente adentro.

As brisas produzem diversas variações diurnas e sazonais na precipitação, tornando-se importante fator para o aprimoramento da modelagem numérica e para a previsão de curto prazo (Birch et al., 2015). Ademais, o aprimoramento observacional da dinâmica das brisas na Amazônia torna-se essencial para que seja feita melhor caracterização diurna da precipitação. Assim, este trabalho tem por finalidade demonstrar a variabilidade atmosférica da precipitação na mesoescala, associada com as circulações de BM e BT na região nordeste do Pará.

\section{MATERIAIS E MÉTODOS}

O sítio experimental está localizado em uma área de cultivo de mangueira, apresentando aproximadamente 25,8 hectares, posicionado na região de Cuiarana (Figura 1) (0³9' 49,72" S, 47 17’ 03,41” O, 17 m), município de Salinópolis, no nordeste do estado do Pará. O sítio é pertencente à Universidade Federal do Pará (UFPA) e à Universidade Federal Rural da Amazônia (UFRA).

Foram utilizados dados de velocidade do vento $(\mathrm{m} / \mathrm{s})$, direção (graus) e precipitação $(\mathrm{mm})$, coletados em frequência contada de 10 em 10 minutos, a partir da torre micrometeorológica, localizada no sítio pertencente ao Projeto Large Scale Biosphere-Atmosphere Experiment in Amazonia (LBA), levando-se em consideração o período 


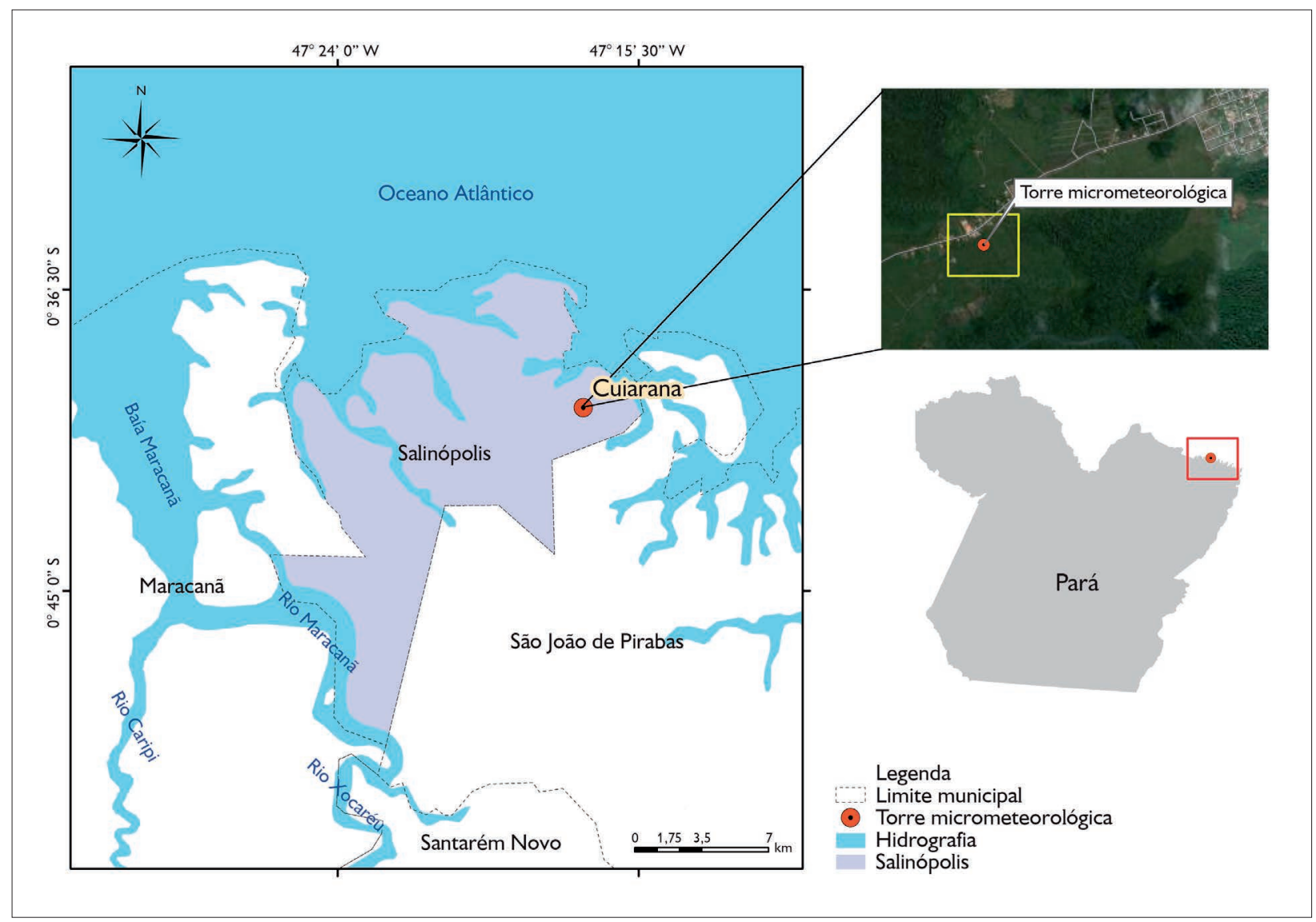

Figura 1. Localização geográfica do sítio experimental de Cuiarana.

de outubro de 2010 até os dias atuais. Em seguida, foram selecionados dois meses da série de dados, um para representar o período chuvoso (março de 2011 a 2013) e outro para o período menos chuvoso (agosto de 2011 a 2013) do nordeste paraense, tendo sido comparadas as características de direção e de velocidade horária do vento, associando-as à precipitação horária. Apenas esses meses foram selecionados para este estudo devido à irregularidade temporal causada por ausências e erros na coleta dos dados.

Para a análise da chuva fracionada, foi utilizada a seguinte fórmula, baseada em Kousky (1980):

$$
C F=\frac{\sum_{i}^{n} P R P}{T}
$$

Onde:

$C F=$ chuva fracionada

$n, i=$ intervalo a ser analisado

$P R P=$ acumulado de precipitação

$T=$ total (mensal)

Para a análise da direção e da velocidade do vento, foi utilizado o software estatístico R, por meio do qual se decompôs o vento em componente zonal (u) e meridional (v). Gerou-se uma sub-rotina para o cálculo das médias horárias mensais, necessárias à caracterização da direção e da velocidade do vento em superfície.

Para o estudo de caso, excepcionalmente, foi selecionado um evento observacional de brisa terrestre, marítima e LI referente ao dia 11 de junho de 2010. A escolha do evento está relacionada com o período menos

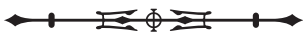


chuvoso da região, ou seja, de menor atuação dos sistemas meteorológicos precipitantes com grande escala e que apresenta possibilidade de maior observação dos efeitos locais (Molion, 1987). Para este evento, foram utilizados dados horários da estação automática do Instituto Nacional de Meteorologia (INMET), localizada no município de Salinópolis. Os dados meteorológicos desta cidade foram adotados no estudo de caso, a fim de se observar a circulação de mesoescala na região. Foram utilizadas imagens de satélite, feitas pelo Centro de Previsão do Tempo e Estudos Climáticos/Instituto Nacional de Pesquisas Espaciais (CPTEC/INPE), para visualização das linhas de nebulosidade associadas às brisas (terrestre e marítima). Esta identificação por imagem de satélite evidenciou melhor aproveitamento quando feita no canal visível. Teixeira (2008) fez uma boa demonstração do uso de imagens de satélite para a detecção da nebulosidade relacionada às brisas (ou frente de brisas) no canal visível e infravermelho.

Para o cálculo da frequência horária das variáveis meteorológicas referentes à direção e à intensidade do vento, utilizou-se o pacote METeorological Visualisation
Utilities using R for Science and Teaching (METVURST) (Appelhans et al., 2013). A mesma metodologia foi utilizada em Appelhans et al. (2013), para fazer a climatologia da frequência da direção e da velocidade do vento em Nova Zelândia.

\section{RESULTADOS E DISCUSSÃO}

\section{ASPECTOS SAZONAIS}

Na Figura 2A, observa-se uma variação significativa no sentido do vento no mês de março, mudando de leste $(\mathrm{E})$, para sudeste $(\mathrm{SE})$, no horário das 2 às $6 \mathrm{~h}(\mathrm{HL})$. Esta mudança pode estar associada à circulação de BT, que ocorre devido à diferença nos coeficientes térmicos entre a superfície do mar e à da terra, já que, no período noturno, o mar está mais aquecido do que a terra e o deslocamento dos ventos ocorre no sentido da terra para o oceano. No intervalo das 7 às $14 \mathrm{~h}(\mathrm{HL})$, a direção do vento é predominantemente de nordeste (NE), apresentando os maiores valores de velocidade do vento durante o dia. Isto pode caracterizar a atuação da BM,

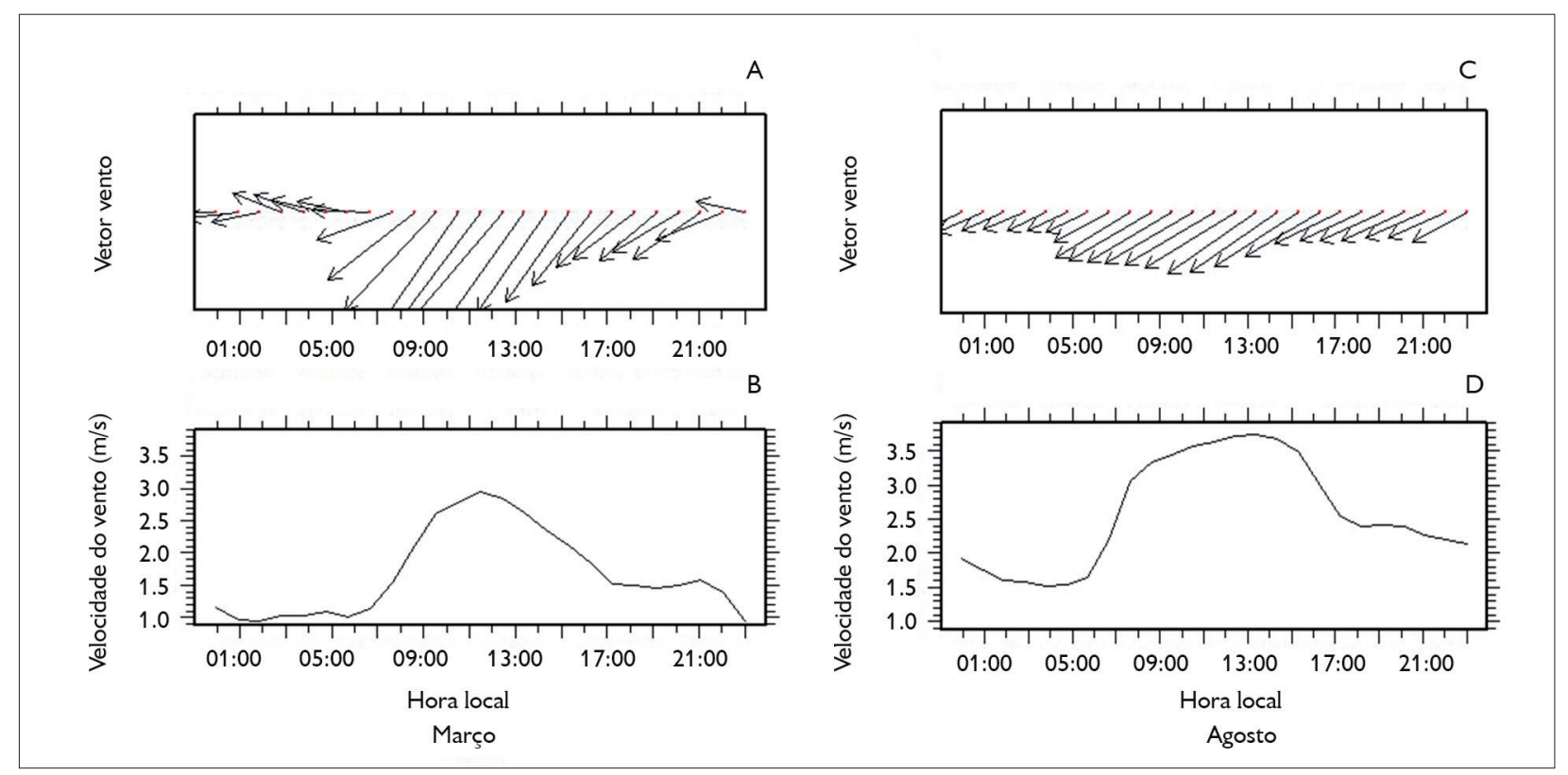

Figura 2. Padrões temporais do ciclo diurno da direção ( $A$ e C) e da velocidade do vento (B e D) na superfície, em Cuiarana, para março e agosto de 2011.

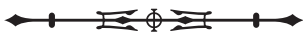


que ocorre devido à diferença térmica entre a terra aquecida e o oceano mais frio, aumentando a velocidade do vento ao longo do dia (Teixeira, 2008).

No mês de agosto (Figura 2C), foi observada pequena variabilidade temporal na direção dos ventos, com predomínio de NE. Isto provavelmente ocorre devido à sobreposição dos alísios sobre as brisas, tornando-os mais predominantes. A velocidade do vento mostra maior intensidade em agosto (Figura 2D) do que em março (Figura 2B), com máximo de 3,7 m.s e de 2,9 m.s. respectivamente. Segundo Germano et al. (2017), a BM no litoral paraense ocorre no mesmo sentido dos alísios, justificando maior magnitude do vento durante os horários da $B M$, entre 11 e $15 \mathrm{~h}(\mathrm{HL})$.

Varejão-Silva (2006) afirma que, em regiões onde os ventos alísios são constantes, como o Norte e Nordeste brasileiros, as brisas quase sempre contribuem para modificar um pouco a direção e a intensidade dos ventos. Dependendo da orientação da costa, a velocidade do vento resultante da superposição alísio-brisa pode ser maior ou menor do que a do alísio. Isso justifica a variabilidade da direção do vento em agosto não ser muito grande no Nordeste paraense.

Foi observado que, no mês de março (Figura 3A), durante as primeiras horas da manhã (2 às $6 \mathrm{~h}-\mathrm{HL}$ ), o vento teve uma pequena variabilidade na direção, porém sem giro significativo na sua direção, não caracterizando BT; o mesmo ocorre no mês de agosto (Figura 3C). Nesse caso, a não caracterização da BT pode estar relacionada com a suavização dos dados de vento, através do cálculo da média entre a velocidade e a direção do vento, observada com fraca intensidade para alguns dias.

A velocidade do vento se intensifica de acordo com os horários de maior temperatura do ar, em razão do aumento do gradiente térmico entre as superfícies da terra e do mar (Simpson, 1994), deslocando o vento da alta pressão para a baixa, chegando à velocidade máxima de $3,0 \mathrm{~m} \cdot \mathrm{s}^{-1}$ às $11 \mathrm{~h}(\mathrm{HL})$ (Figura 3B). Durante o mês de agosto (Figura 3D), a direção do vento se mantém constante, porém, a velocidade aumenta, em virtude da entrada da BM na costa, com ventos de $3,8 \mathrm{~m} \cdot \mathrm{s}^{-1}$ às $13 \mathrm{~h}(\mathrm{HL})$.

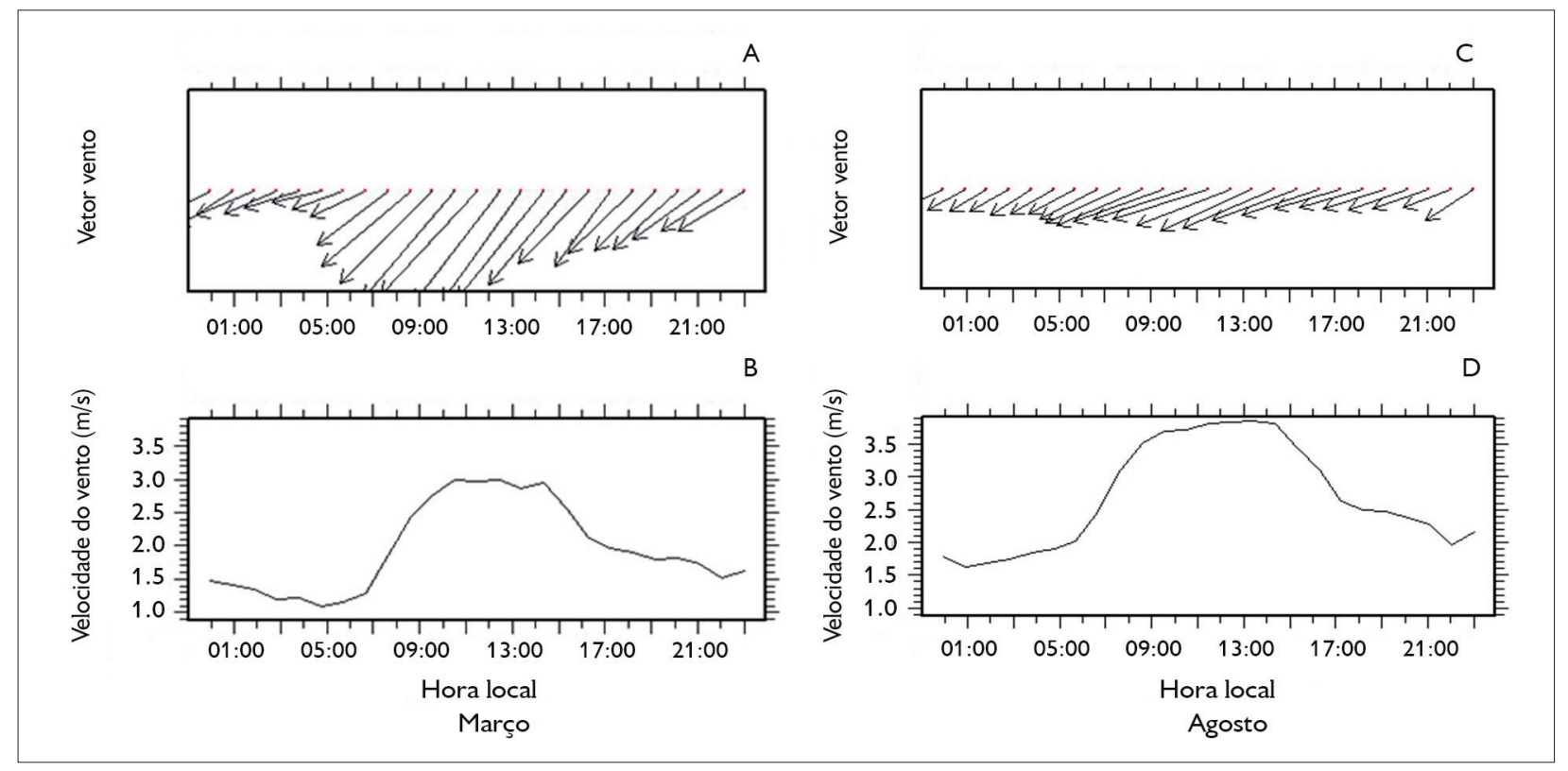

Figura 3. Padrões temporais do ciclo diurno da direção ( $\mathrm{A}$ e C) e da velocidade do vento (B e D) na superfície, em Cuiarana, para março e agosto de 2012.

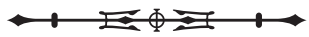


Foi observada pouca variabilidade na direção do vento, tanto em março quanto em agosto de 2013 (Figuras 4A-4C), porém é possível observar a entrada da BM juntamente com o aumento na magnitude do vento em ambos os meses. A velocidade do vento foi maior em agosto (Figura 4D), com máximo de 3,6 m.s $\mathrm{s}^{-1}$ às $13 \mathrm{~h}$, enquanto, em março (Figura 4B), o máximo foi de $3,1 \mathrm{~m} \cdot \mathrm{s}^{-1}$ às $13 \mathrm{~h}(\mathrm{HL})$.

Foi identificada também mudança significativa no padrão dos ventos, tanto na velocidade quanto na magnitude, entre os anos de 2011 a 2013. Não se pode afirmar, neste estudo, que os padrões das bacias dos oceanos Pacífico e Atlântico tropicais possam estar causando variações nas circulações de brisas, no entanto estes resultados geram indícios de que alguns mecanismos de escala maior podem estar provocando variabilidade interanual nas circulações de brisas. Segundo Ferreira \& Silva Mello (2005), a circulação atmosférica sobre a região tropical é fortemente modulada e modificada pelos padrões termodinâmicos sobre as bacias dos oceanos Pacífico e Atlântico tropicais.

Segundo Cohen et al. (1995), as linhas de instabilidade tendem a ocorrer com mais frequência entre abril e agosto, o que converge com os resultados encontrados para ocorrência de BM. As linhas de instabilidade têm uma preferência pela estação seca, quando a convecção diurna sobre a bacia amazônica é menor. Diversos estudos mostram que a circulação de BM serve como gatilho para a formação das linhas de instabilidade (Germano et al., 2017; Cohen et al., 1995; Kousky, 1980).

Observou-se que, no mês de março entre os anos de 2011 a 2013, a precipitação se comporta de forma uniforme (Figuras 5A-5C), devido à presença da nebulosidade relacionada com a zona de convergência intertropical (ZCIT) (Uvo, 1989). Vale ressaltar que a posição latitudinal da ZCIT em relação ao equador modula o regime chuvoso na região amazônica. Por outro lado, durante os meses de agosto, a precipitação ocorre de modo isolado e distribui-se preferencialmente entre o período noturno e o início da manhã. Kousky (1980) sugere que esses horários preferenciais ocorrem em decorrência da convergência entre os fluxos médios superficiais, proveniente do oceano e do continente.

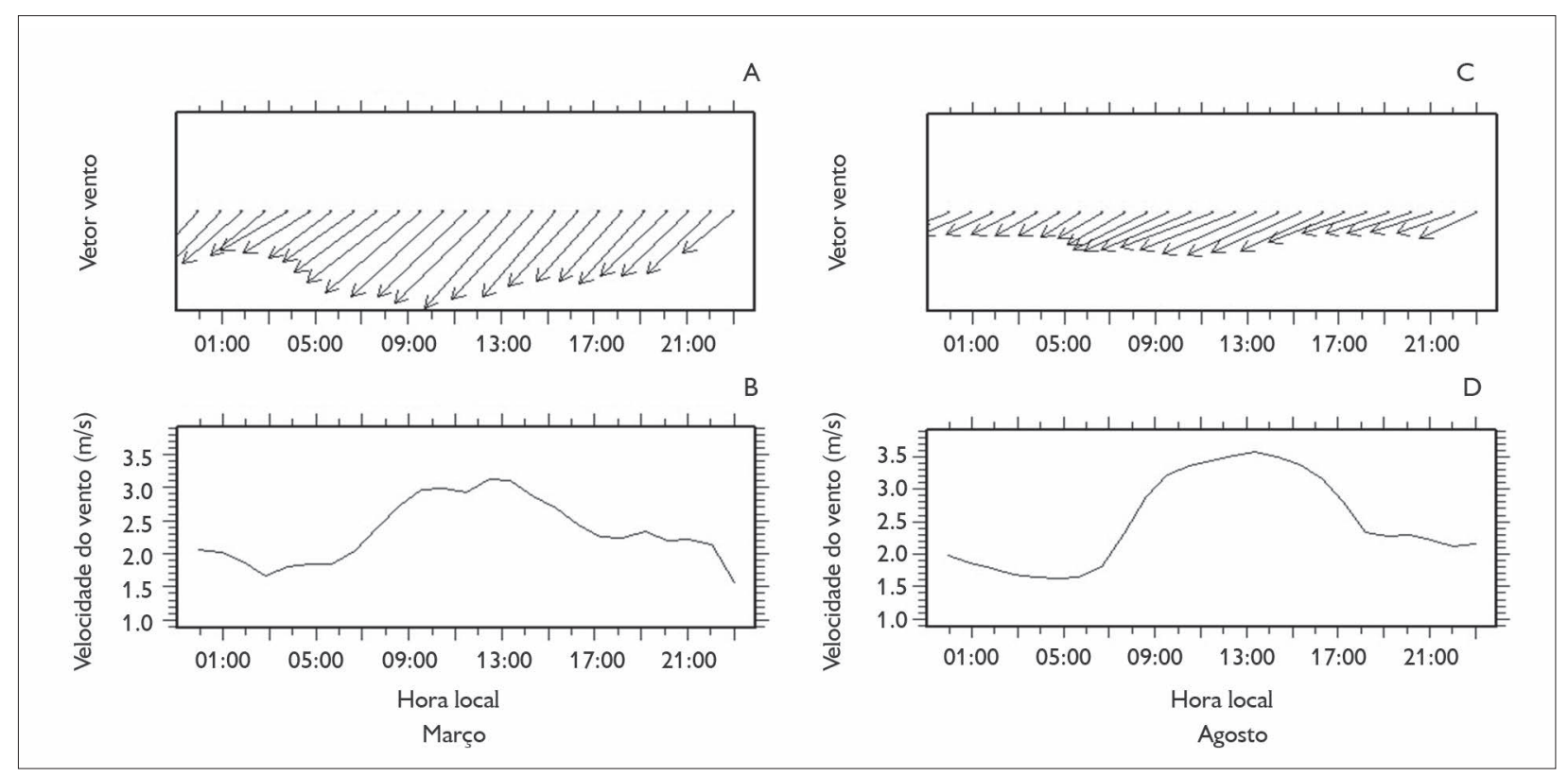

Figura 4. Padrões temporais do ciclo diurno da direção ( $A$ e C) e da velocidade do vento (B e D) na superfície, em Cuiarana, para março e agosto de 2013.

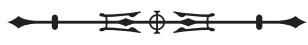




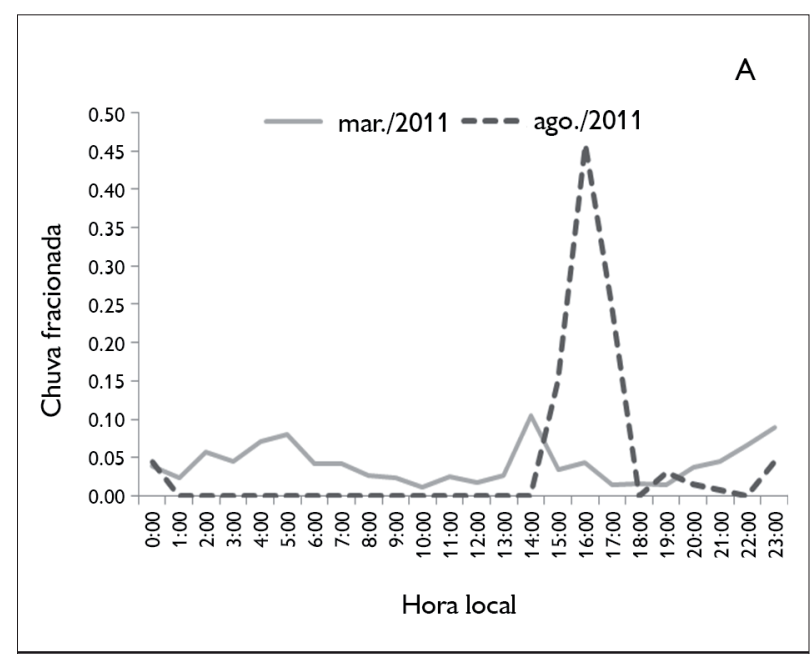

B

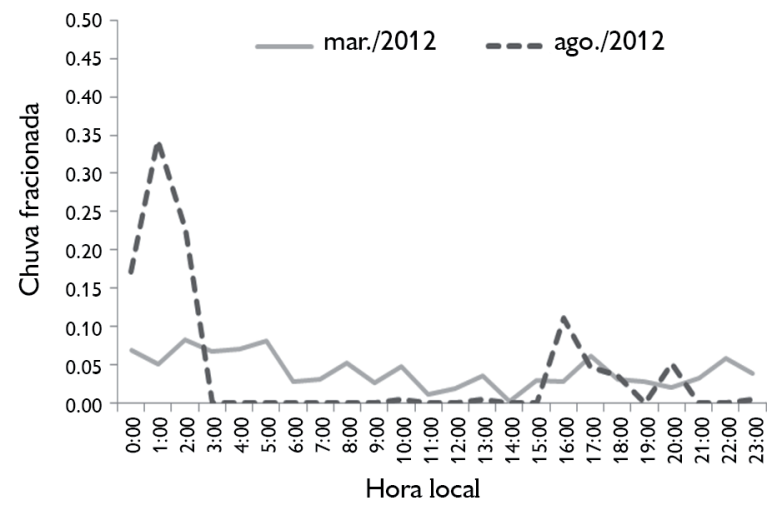

C

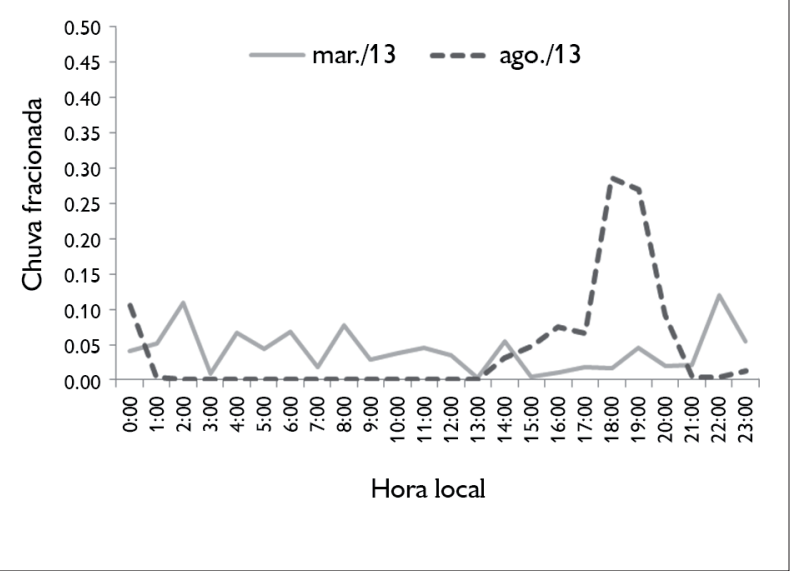

Figura 5. Frequência de chuva por hora para os meses de março e agosto de 2011 (A), 2012 (B) e 2013 (B), em Cuiarana.
Além disso, percebeu-se elevação de ocorrência de chuva no período da tarde, em torno das $18 \mathrm{~h}$, e essa configuração pode estar diretamente relacionada à propagação de chuvas tipicamente de origem locais como sistemas convectivos de mesoescala (Maddox, 1980; Vitorino et al., 1997; Sodré, 2013), associados aos sistemas convectivos circulares ou com linhas de instabilidade (Cohen et al., 1995). Pôde-se notar, ainda, a ocorrência de chuvas na madrugada, em agosto de 2012. Para avaliar essa chuva, esse mês foi analisado independentemente, percebendo-se a presença de BT na madrugada do dia 4 de outubro, acumulando total de 40,64 mm. Observou-se sensível redução no acumulado de precipitação para os meses de agosto, entre os anos de 2011 a 2013.

\section{ESTUDO DE CASO}

Em geral, observou-se que o ciclo diurno da direção do vento para o mês de junho de 2010 apresentou máximo principal de NE, com ocorrência da BM no mesmo sentido dos alísios de nordeste e maior frequência durante $\mathrm{O}$ período de 12 às $21 \mathrm{~h}(\mathrm{HL})$ (Figuras 6A e 6B). A BT pode ser identificada a partir de um máximo secundário na direção de SE a Sul (S), no período da noite e no início da manhã ( 6 às $12 \mathrm{~h}-\mathrm{HL}$ ). Verificou-se que os menores valores da velocidade do vento ocorrem no horário em que estas circulações estão atuando (Figura 6B).

Observou-se que a direção do vento (Figura 6A), durante a madrugada e o início da manhã do dia 11 de junho de 2010, esteve de sul a sudeste (Figuras 7A e 7B), indicando a presença da BT. No entanto, durante o período da tarde, verificou-se variação significativa na direção do vento, apresentando um padrão predominantemente de nordeste, indicando a presença da BM. Foi constatado máximo na velocidade do vento durante o horário de ocorrência da BM, e menores valores foram observados durante a ocorrência da BT.

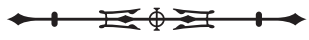




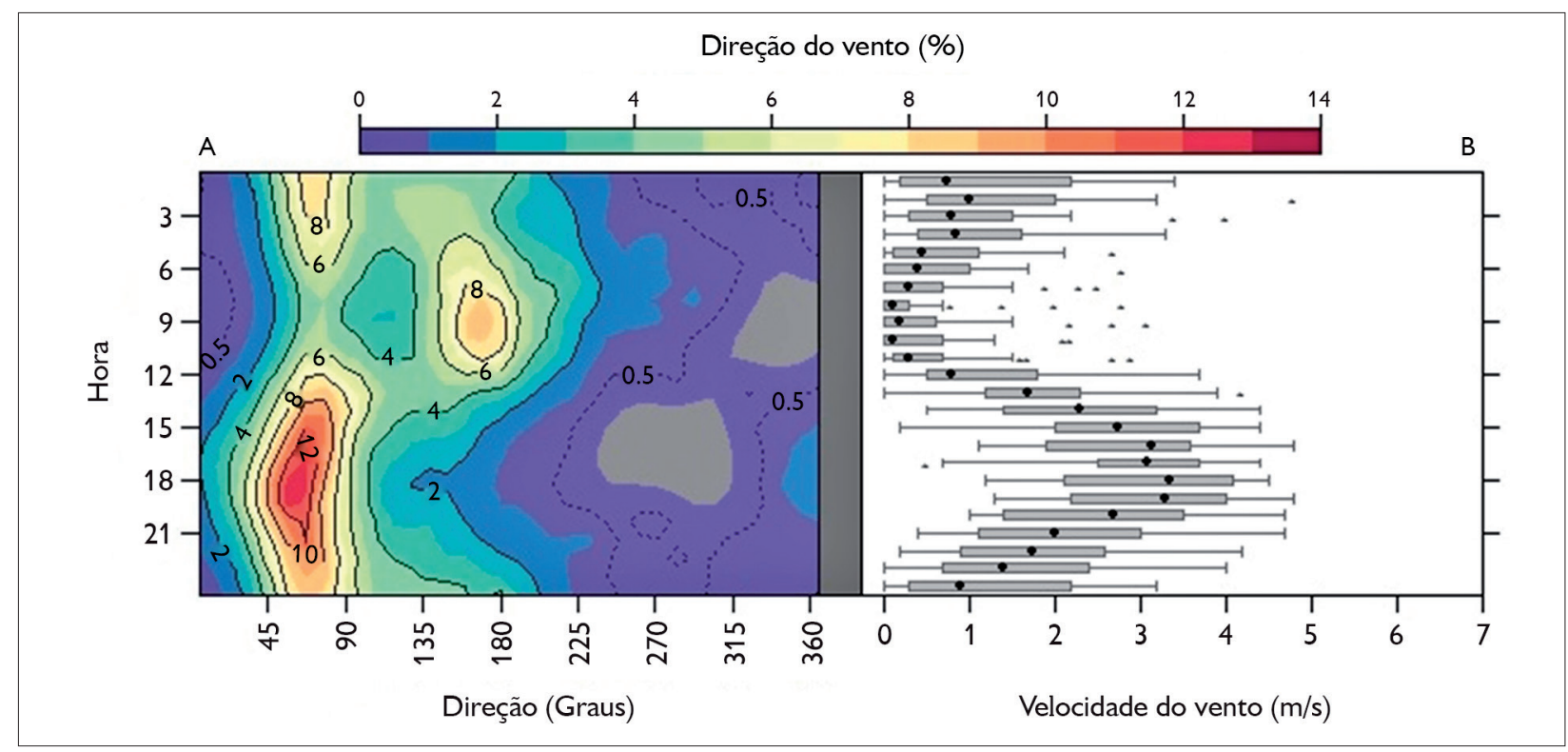

Figura 6. Frequência horária da direção do vento horizontal (A) e velocidade do vento (B) para junho de 2010, em Salinópolis.

Percebeu-se linhas de nebulosidade (linhas tracejadas na Figura 8) sobre o oceano, em decorrência da circulação de brisa terrestre na madrugada do dia 11 de junho de 2010, a qual se propaga a partir do litoral, no sentido do oceano. Segundo Teixeira (2008), é comum observar no litoral arcos convectivos sobre o oceano, em virtude do acoplamento da circulação de brisa terrestre ao fluxo ou escoamento (úmido) médio próximo à costa, resultando em arcos de nuvens em deslocamento para o oceano, favorecendo chuvas mais rápidas e pouco intensas no litoral, durante o período da madrugada e do início da manhã.

Nas imagens de satélite, foi possível perceber a formação de uma LI no litoral (Figura 9), no dia 11 de junho de 2010, a qual teve início às 17 h UTC e término às $6 \mathrm{~h}$ UTC do dia posterior. Vale ressaltar que o horário de formação desta LI ocorre durante a circulação de BM. A diferença térmica entre as superfícies do oceano e terrestre aumenta durante o dia, ocasionando a circulação de BM, que alcança maior intensidade no período da tarde. De acordo com estudos anteriores (Simpson, 1994; Ahrens, 2011; Teixeira, 2008), isto pode favorecer maior transporte de umidade proveniente do oceano para o litoral, vindo a

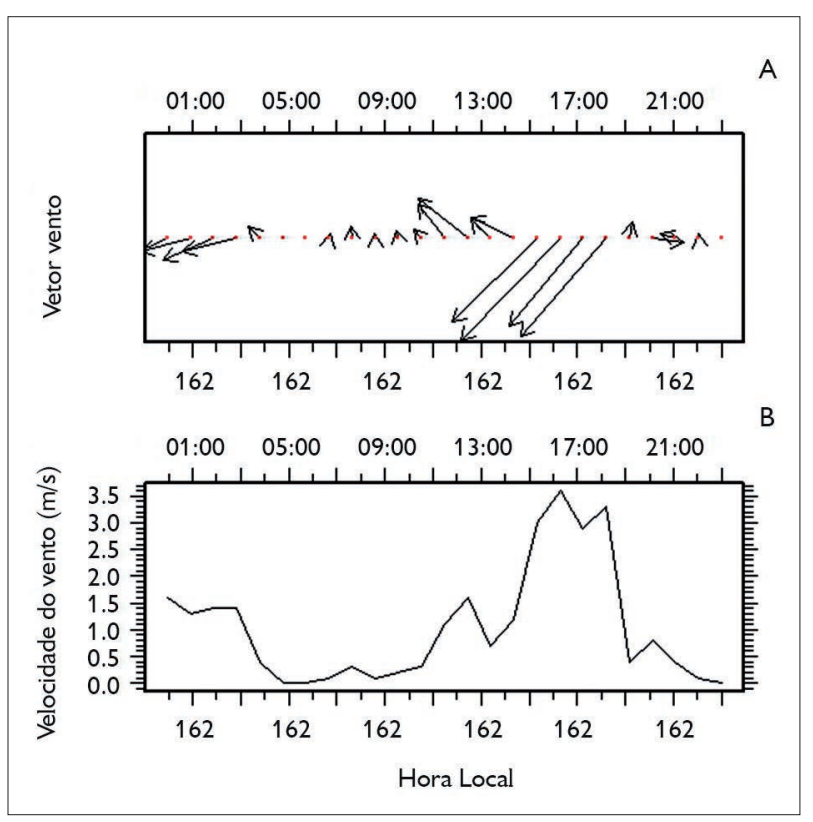

Figura 7. Ciclo diurno da direção (A) e da velocidade (B) do vento em Salinas, para o dia 11 de junho de 2010.

apresentar nuvens mais desenvolvidas, próximo à frente de brisa. Além disso, a formação e a evolução da circulação de BM desenvolvem um papel fundamental na propagação das linhas de instabilidade (Cohen et al., 1995).

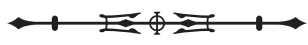




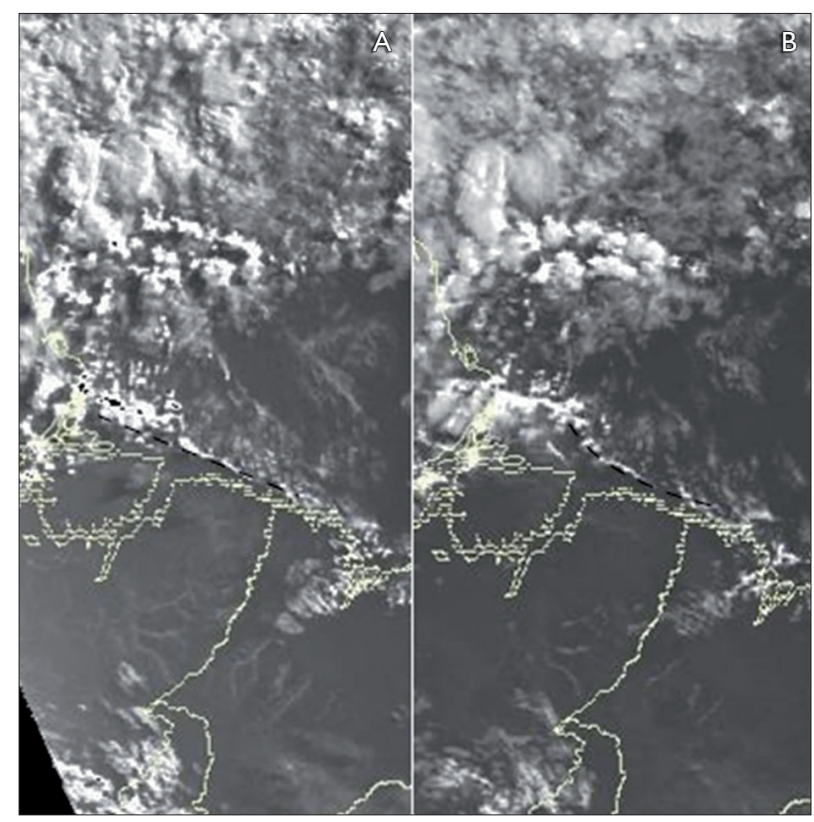

Figura 8. Linha de nebulosidade associada à brisa terrestre. Imagens no canal visível, feitas pelo METEOSAT-9, feitas em 11 de junho de 2010, às 9 h 45 UTC (A) e 11 h UTC (B).

\section{CONCLUSÕES}

Este artigo objetivou principalmente investigar a variabilidade da circulação atmosférica na mesoescala e a sua associação com os eventos de precipitação no litoral do estado do Pará. Para tanto, utilizamos dados de vento horizontal e de precipitação, provenientes de estações automáticas de superfície, juntamente com imagens de satélite.

A circulação de BM apresentou maior intensidade durante os meses de agosto, acompanhada do aumento da velocidade do vento horizontal. Esta circulação teve dominância na direção do vento de nordeste, com intensidade máxima de $3 \mathrm{~m} \cdot \mathrm{s}^{-1}$ no período diurno, das 6 às $18 \mathrm{~h}(\mathrm{HL})$. Por outro lado, a BT mostrou-se melhor caracterizada em março, com intensidade de $1 \mathrm{~m} \cdot \mathrm{s}^{-1}$ na direção sudeste. No entanto, a circulação de BT não foi regular como o foi a de BM.

A precipitação horária local apresentou-se regular durante os meses de março de cada ano. Enquanto, em agosto, observou-se máximo no período da noite, em torno das $18 \mathrm{~h}(\mathrm{HL})$. Além disso, verificou-se a

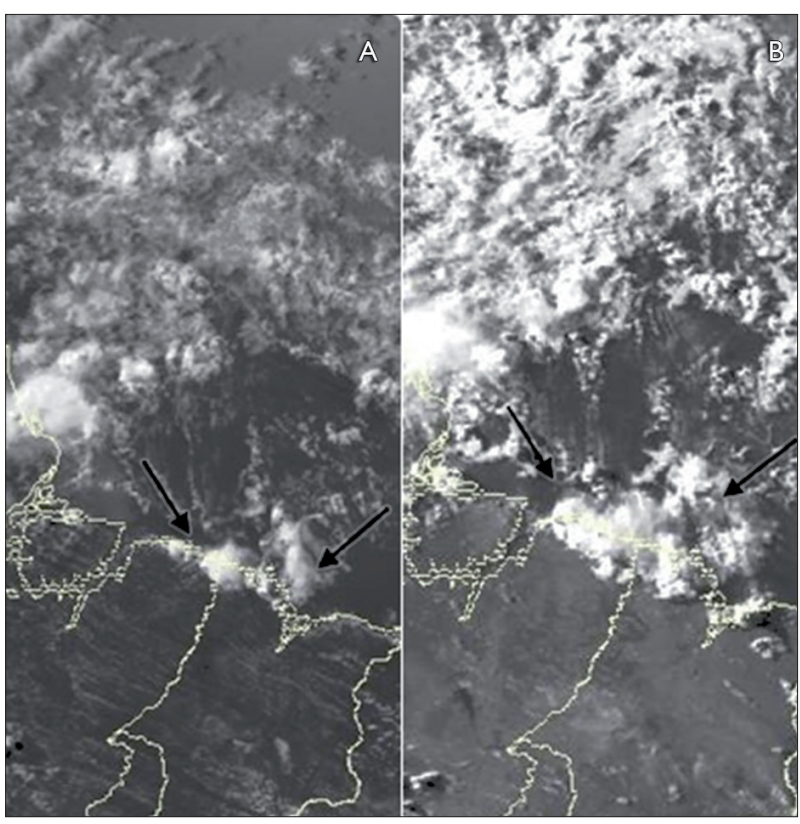

Figura 9. Imagem de satélite feitas pelo METEOSAT-9 no canal visível para o dia 11 de junho 2010, às 18 h 15 UTC (A) e 20 h UTC (B).

formação de LI no mesmo horário de ocorrência da BM, bem como de arcos convectivos sobre o oceano, em decorrência da BT.

Este estudo possibilitou uma investigação mais aprofundada do papel da BM na iniciação das LI, conforme sugestionado por Germano et al. (2017) e em diversas outras publicações. Estudos observacionais como este podem colaborar para o aperfeiçoamento da modelagem numérica referente à região da costa do Pará, em virtude de as brisas afetarem diretamente o ciclo diurno e contribuírem para a climatologia da precipitação na região.

\section{REFERÊNCIAS}

AHRENS, C. D., 2011. Essentials of meteorology: an invitation to the atmosphere. Cengage Learning, Belmont.

APPELHANS, T., A. STURMAN \& P. ZAWAR-REZA, 2013. Synoptic and climatological controls of particulate matter pollution in a Southern Hemisphere coastal city. International Journal of Climatology 33(2): 463-479. DOI: https://doi.org/10.1002/ joc. 3439

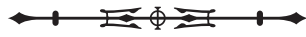


BIRCH, C. E., M. J. ROBERTS, L. GARCIA-CARRERAS, D. ACKERLEY, M. J. REEDER, A. P. LOCK \& R. SCHIEMANN, 2015. Sea-breeze dynamics and convection initiation: the influence of convective parameterization in weather and climate model biases. Journal of Climate 28(20): 8093-8108. DOI: https://doi.org/10.1175/ JCLI-D-14-00850.1.

COHEN, J. C., M. A. SILVA DIAS \& C. A. NOBRE, 1995. Environmental conditions associated with Amazonian squall lines: a case study. Monthly Weather Review 123(11): 3163-3174. DOI: https://doi.org/10.1175/1520-0493(1995)123<3163:ECAWAS > 2 .0.CO;2.

FERREIRA, A. G. \& N. G. SILVA MELLO, 2005. Principais sistemas atmosféricos atuantes sobre a região Nordeste do Brasil e a influência dos oceanos Pacífico e Atlântico no clima da região. Revista Brasileira de Climatologia 1(1): 15-28.

GERMANO, M. F., M. I. VITORINO, J. C. P. COHEN, G. B. COSTA, J. I. D. O. SOUTO, M. T. C. REBELO \& A. M. L. SOUSA, 2017. Analysis of the breeze circulations in Eastern Amazon: an observational study. Atmospheric Science Letters 18(2): 67-75. DOI: https://doi.org/10.1002/asl.726.

HAURWITZ, B., 1947. Comments on the sea-breeze circulation. Journal of Meteorology 4(1): 1-8.

JANOWIAK, J. E., V. E. KOUSKY \& R. J. JOYCE, 2005. Diurnal cycle of precipitation determined from the CMORPH high spatial and temporal resolution global precipitation analyses. Journal of Geophysical Research: Atmospheres 110(D23): 1-18. DOI: https:// doi.org/10.1029/2005JD006156.

KOUSKY, V. E., 1980. Diurnal rainfall variation in northeast Brazil. Monthly Weather Review 108(4): 488-498. DOI: https://doi. org/10.1175/1520-0493(1980)108<0488:DRVINB > 2.0.CO;2.

MADDOX, R. A., 1980. Meoscale convective complexes. Bulletin of the American Meteorological Society 61(11): 1374-1387. DOI: https://doi.org/10.1175/1520-0477(1980)061<1374:MCC $>2.0 . \mathrm{CO} ; 2$
MOLION, L. C. B., 1987. Climatologia dinâmica da região amazônica: mecanismos de precipitação. Revista Brasileira de Meteorologia 2(1): 107-117.

NEGRI, A. J., R. F. ADLER, E. J. NELKIN \& G. J. HUFFMAN, 1994. Regional rainfall climatologies derived from Special Sensor Microwave Imager (SSM/I) data. Bulletin of the American Meteorological Society 75(7): 1165-1182. DOI: https://doi.org/10.1175/15200477(1994)075<1165:RRCDFS > 2.0.CO;2.

SIMPSON, J. E., 1994. Sea breeze and local winds. Cambridge University Press, Cambridge.

SODRÉ, G. R., 2013. Estudo da convecção de mesoescala em diferentes superfícies na Amazônia oriental. Dissertação (Mestrado em Ciências Ambientais) - Universidade Federal do Pará, Belém.

SUN, W. Y. \& I. ORLANSKI, 1981. Large mesoscale convection and sea breeze circulation. Part I: Linear stability analysis. Journal of the Atmospheric Sciences 38(8): 1675-1693. DOI: https://doi. org/10.1175/1520-0469(1981)038<1675:LMCASB > 2.0.CO;2.

TEIXEIRA, R. F. B., 2008. O fenômeno da brisa e sua relação com a chuva sobre Fortaleza-CE. Revista Brasileira de Meteorologia 23(3): 282-291. DOI: http://dx.doi.org/10.1590/S010277862008000300003

UVO, C. R. B., 1989. A Zona de Convergência Intertropical (ZCIT) e sua relação com a precipitação da região Norte do Nordeste Brasileiro: 1-82. Dissertação (Mestrado em Meteorologia) - Instituto Nacional de Pesquisas Espaciais, São José dos Campos.

VAREJÃO-SILVA, M. A., 2006. Meteorologia e climatologia. Versão digital, 2. Recife. Disponível em: <http://www.icat.ufal. br/laboratorio/clima/data/uploads/pdf/METEOROLOGIA E CLIMATOLOGIA_VD2_Mar_2006.pdf >. Acesso em: agosto $2 \overline{013}$.

VITORINO, M. I., M. E. S. SILVA \&J. M. B. ALVES, 1997. Classificação de sistemas convectivos de mesoescala no setor norte do Nordeste brasileiro. Revista Brasileira de Meteorologia 12(1): 21-32.

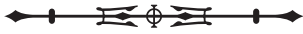

